\title{
POSITION-DEPENDENT VENTRICULAR TACHYCARDIA RELATED TO PERIPHERALLY INSERTED CENTRAL VENOUS CATHETER
}

\author{
Paulino Alvarez, M.D.; Paul Schurmann, M.D.; Melanie Smith, R.N.; Miguel Valderrábano, M.D.; \\ C. Huie Lin M.D., Ph.D. \\ Houston Methodist DeBakey Heart \& Vascular Center, Houston Methodist Hospital, Houston, Texas
}

\begin{abstract}
We report a case of a 51-year-old male who developed frequent nonsustained episodes of monomorphic ventricular tachycardia after being diagnosed with bioprosthetic aortic valve endocarditis and treated with intravenous antibiotics. A peripherally inserted central venous catheter (PICC) had been placed without complication less than 24 hours prior to the episodes. Ventricular tachycardia (VT) occurred during the night, while sleeping, when he assumed a right lateral decubitus position with abduction of the right arm and placement of the forearm under his head. VT occurred repeatedly when such position was assumed again upon request, and it would terminate immediately when sitting upright. The PICC was repositioned in the superior vena cava without further VT. He was discharged home the same day and underwent successful aortic valve replacement 2 months later. Position-dependent VT related to PICC requires careful history taking and PICC repositioning to make the diagnosis. X-ray in different patient positions during PICC placement can be considered to evaluate for ventricular migration.
\end{abstract}

\section{Introduction}

Peripheral inserted central catheter (PICC) is widely used in clinical practice with overall excellent safety. Position-dependent ventricular tachycardia (VT) is a rare complication from PICC but has a good prognosis if treated in a timely manner. Special attention to clinical clues should be applied early on in the diagnostic evaluation. We report a typical presentation of position-dependent VT to reinforce awareness of this entity.

\section{Case Report}

A 51-year-old man was admitted to our hospital after several weeks of weight loss (approximately 40 pounds), dyspnea on exertion (New York Heart Association functional class II), and anemia. He had a history of aortic dissection status post repair and had undergone aortic valve replacement with a bioprosthetic valve 6 years prior. The patient was diagnosed with subacute bioprosthetic aortic valve endocarditis and aortic root abscess due to Lactococcus garvieae, and antibiotic therapy was started.

The night before discharge, he developed multiple episodes of monomorphic nonsustained VT. The morning was uneventful with no new episodes of VT. The patient was asymptomatic for palpitations or chest pain, and vital signs and electrolytes were within normal limits. His electrocardiogram (ECG) on admission showed sinus rhythm and $\mathrm{Q}$ waves in the inferior leads (Figure $1 \mathrm{~A}$ ). Upon further evaluation, it was found that a PICC had been inserted less than 24 hours earlier with the tip located in the upper right atrium (Figure 1 B). We asked the patient to show us how he sleeps, and he assumed a fetal position with abduction of the right arm and placement of the forearm under his head. He promptly developed palpitations and VT that resolved immediately when he sat up again (Figure $1 \mathrm{C}$ ). The PICC line was withdrawn $3 \mathrm{~cm}$, and the patient had no further episodes of VT in his sleeping position. Post-repositioning ECG was unremarkable (Figure $1 \mathrm{D}$ ). Telemetry monitoring was continued for 8 additional hours with no evidence of VT. The patient was discharged the same day to complete outpatient parenteral antibiotic treatment with vancomycin and underwent aortic root abscess debridement and aortic valve replacement 1 month later.

\section{Discussion}

First reported in the year 2000, position-dependent VT related to PICC is a well-described but potentially underreported phenomenon. ${ }^{3}$ In our patient, catheter-induced VT showed regular QRS and monomorphic morphology. The right inferior wall of the right ventricle was likely the location where the tip of the catheter was irritating the myocardium. This was supported by electrocardiographic monitoring showing a left bundle branch block (LBBB) morphology in $\mathrm{V}_{1}$, a positive QRS in lead augmented vector left, and a negative QRS in the inferior leads (Figure $1 \mathrm{~B}$ ).

Use of PICC has expanded since it was first described in 1975, and approximately 3,000 catheters are inserted annually at our hospital. $^{5}$ This increased use is likely because PICC has easy bedside placement, no risk of pneumothorax, minimal risk of bleeding, and long-term durability. Caudal migration of PICC catheter tip with arm abduction has been previously described, especially in catheters located in the right arm. The magnitude of this displacement is $21 \mathrm{~mm}$ with a range between $2 \mathrm{~mm}$ to $53 \mathrm{~mm} .{ }^{6}$ Controversy exists over optimal positioning of the PICC catheter tip; some argue that placement in the SVC reduces catheter-induced arrhythmias, while positioning in the upper right atrium decreases the risk of displacement. ${ }^{2}$ From a practical standpoint, one possible approach would be to ask the patient to demonstrate their usual activities, including sleeping, to evaluate if the position triggers cardiac arrhythmias. $\mathrm{X}$-ray in different patient positions during PICC placement may be considered to evaluate for ventricular migration.

In addition to PICC and central venous catheters, abandoned pacemaker leads have been reported to cause position-dependent VT. $^{7}$

Approximately $2.5 \%$ of patients with endocarditis will develop acute coronary syndrome related to coronary artery compression that results from periannular complications or coronary arterial 


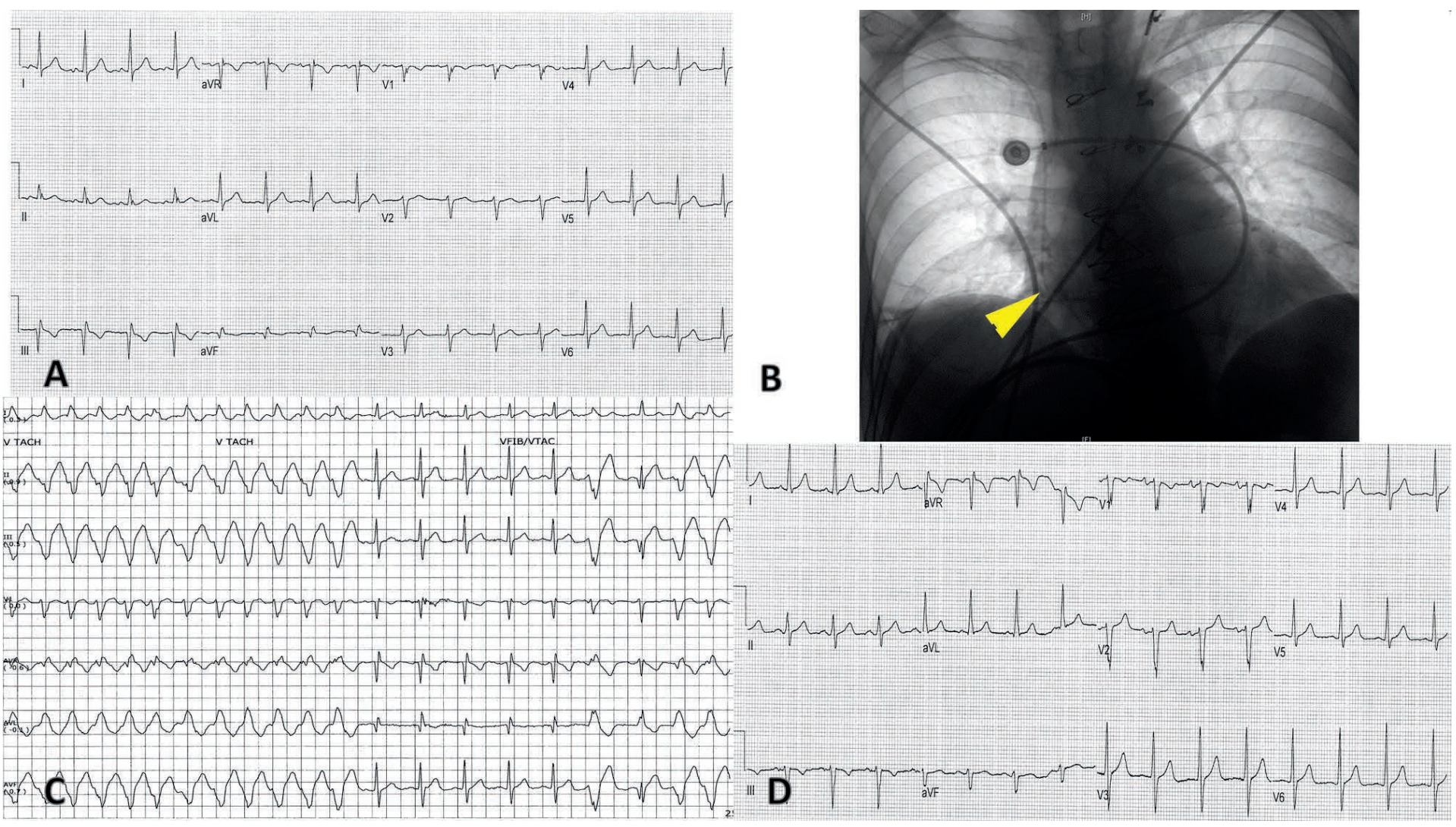

Figure 1. (A) Baseline electrocardiogram (ECG) of patient. (B) Chest X-ray after initial peripherally inserted central catheter (PICC) line placement. Course of PICC line to floor of right atrium can be seen (yellow arrowhead). (C) Telemetry strip of runs of mechanical induction of nonsustained ventricular tachycardia by PICC line during patient positioning. (D) Sinus rhythm ECG after withdrawal of PICC line to superior vena cava-right atrium junction.

embolic events. ${ }^{8}$ Of those $2.5 \%$, a small fraction will present with VT. In our patient, a resting normal ECG, the absence of coronary involvement on cardiac magnetic resonance imaging and coronary angiography, the reproducibility of the VT, and termination of the arrhythmia after changing position excluded the diagnosis of acute coronary syndrome.

\section{Conclusion}

Position-dependent VT related to PICC should be suspected in the appropriate clinical setting. Careful history taking, eliciting maneuvers, and resolution of the arrhythmia with change in position are important to make the diagnosis.

Conflict of Interest Disclosure: The authors have completed and submitted the Methodist DeBakey Cardiovascular Journal Conflict of Interest Statement and none were reported.

Keywords: catheter, ventricular tachycardia, cardiac arrhythmia, positional

\section{References}

1. Gupta AK, Thakur RK. Wide QRS complex tachycardias. Med Clin North Am. 2001;85(2):245-66, ix-X.
2. Vesely TM. Central venous catheter tip position: a continuing controversy. J Vasc Interv Radiol. 2003 May; 14(5):527-34.

3. Bivins MH, Callahan MJ. Position-dependent ventricular tachycardia related to a peripherally inserted central catheter. Mayo Clin Proc. 2000 Apr;75(4):414-6.

4. Hacking MB, Brown J, Chisholm DG. Position dependent ventricular tachycardia in two children with peripherally inserted central catheters (PICCs). Paediatr Anaesth. 2003 Jul;13(6):527-9.

5. Hoshal VL Jr. Total intravenous nutrition with peripherally inserted silicone elastomer central venous catheters. Arch Surg. 1975 May;110(5):644-6.

6. Forauer AR, Alonzo M. Change in peripherally inserted central catheter tip position with abduction and adduction of the upper extremity. J Vasc Interv Radiol. 2000 Nov-Dec;11(10):1315-8.

7. Datta G, Sarkar A, Haque A. An uncommon ventricular tachycardia due to inactive PPM lead. ISRN Cardiol. 2011;2011:232648. Epub 2011 Apr 14.

8. Manzano MC, Vilacosta I, San Roman JA, et al. Acute coronary syndrome in infective endocarditis. Rev Esp Cardiol. 2007 Jan;60(1):24-31. 